\title{
Autoconceito Profissional e Suporte à Transferência e Impacto do Treinamento no Trabalho
}

\author{
Natasha Tamayo \\ Gardênia da Silva Abbad
}

\begin{abstract}
RESUMO
Esta pesquisa investigou o relacionamento entre autoconceito profissional, suporte à transferência e impacto do treinamento no trabalho. As amostras de participantes (modelo 1-auto-avaliação de Impacto como variável critério, $\mathrm{N}=117$; modelo 2-heteroavaliação como variável critério, $\mathrm{N}=$ 100) foi obtida junto a duas organizações do Distrito Federal. A coleta de dados se deu em dois momentos distintos: no primeiro dia de treinamento, quando era aplicada a escala de Autoconceito Profissional e três meses após o término do curso, quando eram aplicados os instrumentos de Impacto e Suporte na amostra de participantes e de suas respectivas chefias. Todos os instrumentos foram validados estatisticamente. Somente Suporte Psicossocial percebido pelo treinando $\left(\mathrm{sr}^{2}=\right.$ $0,25)$ e Suporte Material percebido pela chefia $\left(\mathrm{sr}^{2}=0,16\right)$ contribuíram, respectivamente, na explicação da variabilidade de auto e heteroavaliação de Impacto do Treinamento. Esses resultados corroboram estudos nacionais e estrangeiros no que se refere ao poder explicativo de Suporte Psicossocial sobre os níveis de Impacto. O Autoconceito Profissional não foi preditor de Impacto de Treinamento. Porém, essa variável não deve ser descartada dos modelos de avaliação, pois poderá explicar o impacto de programas de educação e encarreiramento sobre o perfil da clientela.
\end{abstract}

Palavras-chave: autoconceito profissional; suporte à transferência; impacto do treinamento no trabalho; avaliação de treinamento.

\begin{abstract}
This research investigated the relationship between professional self-concept, support to transfer of training and training impact at work. The samples of participants (model 1 - self-assessment as the dependent variable, $\mathrm{N}=117$; model 2 - hetero-assessment as the dependent variable, $\mathrm{N}=100$ ) was obtained from two Brazilian federal government organizations. The data were collected at two distinct moments: on the first day of training, when the Professional Self-Concept scale was administered to the participants, and three months after the training, when the scales of Impact and Support were administered to the trainees and their respective supervisors. All the instruments were statistically validated. Only Psychosocial Support perceived by the trainees $\left(\mathrm{sr}^{2}=0,25\right)$ and Material Support perceived by the supervisors $\left(\mathrm{sr}^{2}=0,16\right)$ contributed, respectively, to the explanation of the variability of self and hetero-assessment of Training Impact. These results corroborated other national and international studies concerning the predictive power of Psychosocial Support over the Impact level. Self-Concept did not predict Impact of Training. However, this variable should not be discarded from the evaluation models, since it could explain the impact of educational programs and career systems over the individual competence profile.
\end{abstract}

Key words: professional self-concept; support to transfer of training; training impact at work; training assessment. 


\section{INTRODUÇÃO}

Atualmente, as mudanças contextuais advindas do progresso científico e tecnológico vêm alterando o cenário mundial e afetando substancialmente as instituições. Em função da globalização, as tendências ambientais das últimas décadas apontam para um cenário caracterizado por mudanças tecnológicas, alta complexidade, velocidade das informações, acirramento de competitividade empresarial, inovação e crescente exigência do consumidor.

Buscando acompanhar a evolução do conhecimento científico, desenvolvimento tecnológico e modernização do setor produtivo, as organizações têm optado por contratar pessoal qualificado e experiente. Como nem sempre é possível encontrar profissionais com o perfil esperado, estas não têm outra alternativa a não ser treinar seus empregados para capacitá-los e poder sustentar-se no mercado competitivo em que se encontram. Este cenário vêm transformando a área de Treinamento e Desenvolvimento (T\&D) num importante instrumento que auxilia organizações e funcionários a adquirirem novas habilidades, conhecimentos, atitudes e comportamentos, podendo, assim, responder às novas exigências e demandas dos postos de trabalho de maneira mais eficaz e eficiente.

As organizações vêm investindo muito em treinamento de pessoal. Porém, na maioria dos casos, este investimento é feito às cegas, ou seja, os treinamentos são oferecidos sem um estudo criterioso prévio das necessidades de treinamento, podendo resultar numa perda de tempo e dinheiro. Hoje em dia, são poucas as organizações que investem em um sistema de treinamento próprio; a maioria compra os cursos em pacotes prontos que nem sempre são adequados à sua realidade. Além disso, muitos treinamentos são oferecidos, sem uma avaliação de sua eficácia e eficiência em termos de impacto no trabalho.

Por essas razões, é essencial a produção de conhecimentos na área de avaliação de treinamento, pois o aperfeiçoamento do sistema depende fundamentalmente dos resultados destas avaliações. A produção científica tem contribuído bastante para que avanços teóricos na área fossem obtidos. Contudo, os conhecimentos acumulados apontam para a necessidade de ampliação do foco de estudo, associando a pesquisa sobre eficácia de treinamento a variáveis individuais. Nesse estudo, investigou-se o relacionamento entre Autoconceito Profissional, Suporte à Transferência e Impacto do Treinamento no Trabalho. 


\section{Treinamento e Desenvolvimento}

Vargas (1996) define Treinamento e Desenvolvimento como a aquisição sistemática de conhecimentos capazes de provocar, a curto ou longo prazo, mudança na maneira de ser e de pensar do indivíduo, por meio da internalização de novos conceitos, valores ou normas e da aprendizagem de novas habilidades. Nadler (1984) faz distinção entre os termos treinamento, desenvolvimento e educação, freqüentemente entendidos como sinônimos, por fazerem referência a práticas organizacionais direcionadas ao aumento da aprendizagem. Treinamento é definido como os esforços das organizações para disponibilizar situações de aprendizagem que propiciem melhoria de desempenho no trabalho. Educação refere-se às estratégias de aprendizagem desenvolvidas para preparar o indivíduo para um trabalho diferente do atual em futuro próximo. Finalmente, o desenvolvimento é o conjunto de ações que promove o crescimento individual, não estando necessariamente relacionado ao trabalho atual ou futuro. Segundo Bastos (1991), uma das maneiras de diferenciar treinamento dos demais conceitos apresentados é pelos critérios da intencionalidade em produzir melhorias de desempenho e do controle exercido pela organização sobre o processo de treinamento.

Borges-Andrade (1982) entende treinamento de pessoal como um sistema composto de três elementos: avaliação de necessidades, planejamento e avaliação. Devido à exigência de rápida aprendizagem e aos altos investimentos em treinamento, a avaliação passou a ser uma ação relevante com finalidade de aumentar a eficiência e eficácia dos sistemas instrucionais. Para Borges-Andrade (1982), avaliação de treinamento é um conjunto de atividades, métodos e princípios utilizados para a validação de informações acerca da efetividade de um dado sistema. A avaliação representa importante papel, uma vez que é o principal responsável pela retroalimentação do sistema instrucional.

Em função do cenário de globalização, as organizações têm feito altos investimentos em cursos de capacitação e concentrado esforços para responder a questões referentes à eficácia e eficiência de seus treinamentos. Desta forma, modelos de avaliação de treinamento têm sido criados para identificar variáveis preditoras da eficácia e eficiência de treinamentos. Os trabalhos pioneiros são os de Kirkpatrick (1976) e Hamblin (1978). Segundo a abordagem destes autores, a execução dos sistemas de treinamento estaria centrada em cinco níveis de análise: reação, aprendizagem, comportamento no cargo, mudança organizacional e valor final. Eles sugerem que estes níveis mantêm entre si um relacionamento positivo e significativo. Entretanto pesquisas como as de Alliger e Janak (1989) e Tannenbaum e Yukl (1992) não confirmam essas suposições. Assim como Goldstein (1991), eles acreditam que a aprendizagem, apesar de 
ser condição necessária para a transferência ou impacto no trabalho, não é condição suficiente para tal.

A análise da literatura nacional e estrangeira sobre T\&D, realizada por BorgesAndrade e Abbad (1996), confirma esses resultados, indicando que reações favoráveis ao treinamento e aprendizagem não garantem a aplicação, no trabalho, das novas habilidades adquiridas em treinamentos. Verifica-se em diversos estudos (Abbad, 1999; Britto, 1999; Sallorenzo, 2000), que o ambiente pós-treinamento desempenha papel importante na determinação de transferência. Em face dessas incongruências, modelos de avaliação de treinamento mais sofisticados foram propostos visando à identificação das variáveis preditoras de impacto do treinamento no trabalho.

O Modelo de Avaliação Integrado e Somativo (MAIS), elaborado por BorgesAndrade (1982), é talvez um dos mais utilizados em pesquisas nacionais. Além de contemplar variáveis internas dos programas de treinamento, ele prevê a influência de variáveis externas sobre os resultados. Segundo o autor, é a investigação conjunta dessas variáveis que permite fornecer informações precisas sobre como elas estão envolvidas no treinamento, podendo-se assim propor mudanças e melhorias no treinamento e em seus subsistemas.

Com base no Modelo MAIS, Abbad (1999) desenvolveu o Modelo Integrado de Avaliação do Impacto do Treinamento no Trabalho - IMPACT, que analisa o valor preditivo de múltiplas variáveis e integra, em uma única abordagem, a avaliação dos três níveis de avaliação: reação, aprendizagem e impacto. Este modelo é composto de sete variáveis: percepção de suporte organizacional, características de treinamento, características da clientela, reação, aprendizagem, suporte à transferência e impacto do treinamento no trabalho.

Abbad, Gama e Borges-Andrade (2000), em estudo sobre o relacionamento entre os três níveis de avaliação do treinamento, afirmaram que uma avaliação adequada da eficácia de treinamentos não se pode restringir à avaliação das reações, apesar de estas serem geralmente relacionadas com o impacto do treinamento no trabalho. Segundo os autores, há fortes preditores de impacto que pertencem a outros componentes, como suporte organizacional e características da clientela. Com a proposta clássica de Cronbach e Snow (1977) de que a pesquisa deveria dar mais ênfase à investigação de interações de aptidões e tratamentos, e não apenas testar diferentes métodos, tornou-se importantíssima a incorporação de variáveis individuais nos modelos de avaliação.

Partindo dessa demanda, propõe-se, com base no modelo de avaliação IMPACT (Abbad, 1999), um modelo reduzido, apresentado na Figura 1, que subsidiou a 
investigação do relacionamento entre as variáveis Autoconceito Profissional, Suporte à Transferência e Impacto do Treinamento no Trabalho.

\section{Figura 1: Modelo de Avaliação de Impacto do Treinamento no Trabalho}

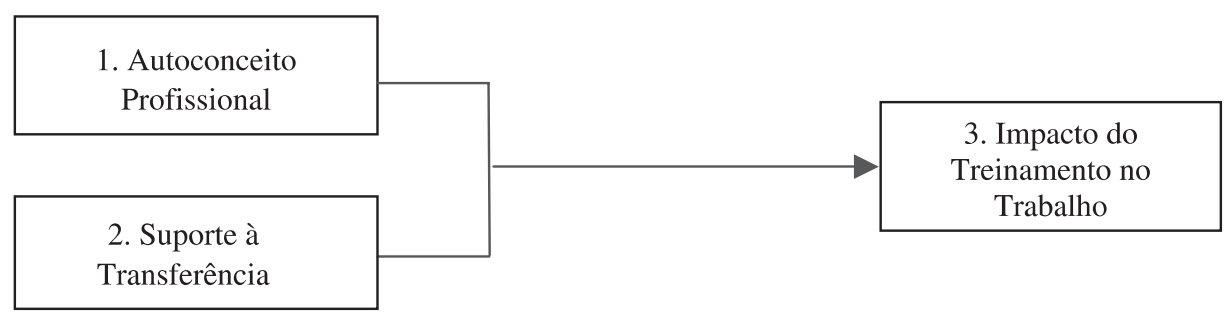

Fonte: Tamayo (2002).

\section{ReVisão de Literatura}

A linha de pesquisa características da clientela estuda o relacionamento entre variáveis individuais da clientela e a eficácia de treinamento. Investigações empíricas em organizações sobre características dos treinandos ainda são limitadas, mas houve um aumento de pesquisas sobre implicações destas no aumento da efetividade dos treinamentos (Tannenbaum \& Yulk, 1992).

Segundo Tannenbaum, Mathieu, Salas e Cannon-Bowers (1991), a eficácia de treinamentos depende, em parte, de características individuais dos treinandos. Pesquisadores se têm interessado pelo estudo da treinabilidade, que tem como objetivo prever, com base em características individuais, se um indivíduo irá completar o treinamento com sucesso (Tannenbaum \& Yulk, 1992). O estudo destas variáveis permite a seleção de pessoas que trarão maiores benefícios à organização após um treinamento em determinada área. Entre elas, as mais estudadas são: motivação, auto-eficácia e locus de controle. Perante o crescente interesse dos pesquisadores na compreensão do relacionamento entre características individuais e a eficácia de treinamentos, sugere-se a introdução de uma nova variável, o Autoconceito Profissional, como possível preditora de Impacto do Treinamento no Trabalho.

O autoconceito é definido por Tamayo (1993) como estrutura cognitiva que organiza as experiências passadas do indivíduo, reais ou imaginárias, controla o processo informativo relacionado consigo mesmo e exerce uma função de auto-regulação.

Shavelson, Hubner e Stanton (1976) identificam alguns aspectos essenciais do 
autoconceito, dentre os quais estão: organização hierárquica, multidimensionalidade, dinamismo e desenvolvimento a partir da interação social com outros significativos. Kihlstrom e Cantor (1984) acreditam que a representação mental do self constituise de um número indefinido e hierarquizado de autoconceitos específicos. Cada um deles representa crenças da pessoa em relação às diferentes situações por ela vivenciadas. A literatura indica que a maioria dos pesquisadores considera o autoconceito um construto multidimensional. L'Ecuyer (1978) acredita que os autores que o percebem como unitário estão apenas aprofundando um de seus aspectos: como, por exemplo, a auto-estima. Por outro lado, os que o abordam como multidimensional lidam com seus diversos componentes e suas interrelações. O autoconceito não se mantém necessariamente estável diante do passar do tempo e aos diferentes acontecimentos presentes na vida do indivíduo, pois sua natureza é dinâmica (Nezlek \& Plesko, 2001). Ele se modifica em função das experiências do sujeito, assim como pode influenciá-las (Cerqueira, 1991). Segundo Sedikides e Skowronski (1997), a formação do autoconceito ocorre por meio da internalização do indivíduo, da forma como as outras pessoas o percebem e o avaliam. Assim, a percepção que o indivíduo tem de si forma-se a partir das representações dos outros, que funcionam como espelho que reflete uma imagem a partir da qual o indivíduo se descobre, se estrutura e se reconhece. Esta relação ocorre durante a interação social por intermédio de comunicação, isto é, olhares, sentimentos, percepções e expectativas (Tamayo, 1982).

Lummertz e Baggio (1986) apresentam uma definição de autoconceito bastante completa. Para eles, o autoconceito consiste em um conjunto de atitudes e crenças interrelacionadas que um indivíduo tem a respeito de si próprio, sendo que estas crenças, produto de sua interação social, são organizadas hierárquica e sistematicamente, formando uma estrutura na qual alguns aspectos são mais resistentes à mudança do que outros e onde uma mudança em qualquer um dos aspectos pode levar a mudanças em outros.

Apesar da percepção e da autopercepção serem aspectos fundamentais para o entendimento do desempenho humano no trabalho, não são encontrados, na literatura, estudos sobre este assunto.

Segundo Costa (1996), o autoconceito revela se o indivíduo está ou não satisfeito consigo mesmo, e esta satisfação ou insatisfação com sua própria pessoa influenciará suas relações com as demais nas organizações nas quais convive (escola, família, trabalho). Assim, entende-se que o nível de autoconceito que um empregado apresenta poderia influenciar seu desempenho no ambiente de trabalho. Porém, em face da multidimensionalidade do construto, a utilização de medidas gerais de autoconceito, que englobam suas diversas facetas, não seria adequada para investigar a autopercepção do indivíduo no trabalho, já que esta representa apenas um dos seus aspectos. Se o objetivo é entender o desempenho 
humano no trabalho, deve-se medir aquela faceta do autoconceito que representa sua satisfação ou insatisfação consigo mesmo apenas no que se refere à sua atuação profissional no trabalho.

Neste estudo, o Autoconceito Profissional é entendido como a percepção que o indivíduo tem de si mesmo em relação ao trabalho e às tarefas que executa. Esta percepção reflete como o indivíduo se percebe em seu trabalho no que diz respeito às suas tarefas, à organização onde trabalha e aos outros significativos na situação de trabalho: chefia, colegas, clientes. Assim, buscou-se investigar de que modo um alto ou baixo Autoconceito Profissional afeta os níveis de Impacto do Treinamento no Trabalho.

No que tange às variáveis situacionais, inúmeras pesquisas mostram que a eficácia de um programa de T\&D pode ser afetada por eventos que ocorrem no ambiente de trabalho do treinando, pois variáveis do contexto organizacional podem controlar os efeitos do treinamento. Saber e querer fazer alguma coisa de modo eficaz não é condição suficiente à transferência positiva de treinamento (Abbad \& Sallorenzo, 2001). A análise da literatura sobre avaliação de treinamento sugere que variáveis do contexto organizacional são fortes preditoras de impacto (Abbad, 1999; Borges-Andrade \& Abbad, 1996; Britto, 1999; Roullier \& Goldstein, 1993; Sallorenzo, 2000; Tannenbaum \& Yukl, 1992).

Conforme Salas e Cannon-Bowers (2001) e Abbad, Pilati e Pantoja (2003), um dos principais responsáveis pela aplicação, no local de trabalho, dos conteúdos de treinamento é o suporte ou clima para transferência. Clima para transferência consiste em situações e conseqüências que inibem ou facilitam a transferência do que foi aprendido no treinamento para o ambiente de trabalho (Roullier \& Goldstein, 1993). Entende-se por suporte à transferência os fatores que podem interferir positiva ou negativamente na aplicação dos novos conhecimentos adquiridos, que podem ir desde a disponibilidade de equipamentos, até os aspectos de suporte da chefia e dos colegas do ex-treinando. A grande diferença entre esses dois conceitos é que o suporte à transferência inclui não só o suporte psicossocial (clima para transferência), mas também o suporte material.

Diversos estudos vêm indicando a importância de variáveis psicossociais de apoio ao uso de novas habilidades no trabalho como preditoras de transferência de treinamento. Roullier e Goldstein (1993), por exemplo, verificaram que clima para transferência foi responsável, em conjunto com o escore de aprendizagem, pela explicação de mais da metade (54\%) dos comportamentos de transferência. De acordo com estes autores, os treinandos que não contam com clima positivo para a transferência de treinamento no trabalho não aplicam no trabalho as habilidades adquiridas em treinamento. 
Segundo Tannenbaum e Yukl (1992), diversos elementos do ambiente póstreinamento podem encorajar, desencorajar ou dificultar a aplicação de novos conhecimentos e habilidades no trabalho. Salas e Cannon-Bowers (2001) acreditam que os acontecimentos que ocorrem após o treinamento são tão importantes quanto os que ocorrem antes e durante o mesmo. Conforme a revisão de literatura realizada por Borges-Andrade e Abbad (1996), existem evidências da influência de fatores organizacionais sobre a transferência de treinamento, entre outros: nível de estímulo e aprovação ao uso de novas tecnologias; qualidade do ambiente de trabalho, bem como dos suportes material, financeiro e gerencial.

A presente pesquisa aborda, entre outras variáveis, a percepção de suporte à transferência. Esta exprime a opinião dos participantes a respeito do nível de apoio fornecido pelo ambiente ao uso eficaz, no trabalho, das novas habilidades adquiridas por meio de eventos instrucionais. Investigou-se nesta pesquisa, o poder preditivo do Suporte à Transferência sobre o Impacto do Treinamento no Trabalho.

No que diz respeito à variável critério do modelo de avaliação de treinamento proposto nesta pesquisa, Impacto do Treinamento no Trabalho, este compreende os conceitos de transferência de treinamento e desempenho no trabalho. A área de T\&D não se interessa apenas pelas medidas de retenção tomadas imediatamente após o treinamento, mas principalmente pelo efeito deste a longo prazo sobre os níveis de desempenho, pela identificação de fatores restritivos e facilitadores do uso das habilidades, capacidades e atitudes adquiridas e das condições necessárias para que tais níveis melhorem. Assim, impacto do treinamento no trabalho é definido como o efeito de longo prazo exercido pelo treinamento nos níveis de desempenho, motivação e atitudes do participante (Abbad et al., 2003). Esta definição não se restringe ao conceito de transferência de treinamento, estando associada também ao conceito de desempenho, pois abrange os diferentes efeitos de treinamento no repertório comportamental do treinando.

O impacto do treinamento no trabalho é uma das principais variáveis critério dos modelos de avaliação de treinamento. Este corresponde ao terceiro nível de avaliação nas abordagens de Kirkpatrick (1976) e Hamblin (1978), desempenho no cargo, definido pelos autores como o efeito do treinamento sobre o desempenho, a motivação e/ou atitudes da clientela. As abordagens de Kirkpatrick (1976) e Hamblin (1978) sugerem um forte relacionamento positivo entre os níveis de reação, aprendizagem, desempenho no cargo (impacto), mudança organizacional e valor final. Porém pesquisas recentes (Alliger \& Janak, 1989; Tannenbaum \& Yukl, 1992) têm mostrado que participantes de treinamento podem apresentar reação favorável ao treinamento e escores satisfatórios nas avaliações de aprendizagem sem, no entanto, aplicar, no trabalho, as novas habilidades adquiridas. Há, portanto, necessidade de oferecer ao empregado treinado as oportunidades para colocar em prática o que aprendeu no treinamento. Reação 
favorável e aprendizagem não são condições suficientes para a transferência ou impacto do treinamento no trabalho.

Dois tipos de medida podem ser utilizados para acessar o impacto do treinamento: 1) a avaliação de impacto em amplitude, cujo interesse reside nos efeitos gerais do evento instrucional sobre o desempenho de tarefas relacionadas diretamente ou não ao conteúdo aprendido no curso; e 2) avaliação de impacto em profundidade, cujo interesse é voltado para o efeito do treinamento em tarefas estritamente relacionadas aos conteúdos específicos ensinados nos cursos (Hamblin, 1978). Nesta pesquisa, adotou-se a avaliação em amplitude, levantando informações sobre freqüência de utilização do conteúdo aprendido, melhoria da qualidade do desempenho de tarefas e diminuição de erros na execução das tarefas.

O principal preditor de impacto em amplitude, segundo Sallorenzo (2000), é o suporte psicossocial à transferência. Outros preditores relevantes são: proximidade do conteúdo ensinado no treinamento às situações reais de trabalho, motivação para aprender e aplicar, reação ao treinamento e, em alguns casos, suporte material e aprendizagem.

Os resultados apresentados indicam que é necessário continuar explorando o poder explicativo de variáveis ambientais ou situacionais, como suporte à transferência; e variáveis individuais (motivação, auto-eficácia, entre outras) sobre o impacto do treinamento no trabalho. Diante disto, pretende-se com o presente estudo propor um modelo que inclui, como variáveis antecedentes, a percepção de suporte organizacional e o Autoconceito Profissional.

Nesta pesquisa, a variável critério foi Impacto em Amplitude do Treinamento no Trabalho, definida como a percepção dos participantes e de suas respectivas chefias, sobre os efeitos imediatos produzidos pelo treinamento no que se refere ao desempenho, à motivação e/ou atitudes dos treinandos.

\section{Delimitação do Problema de Pesquisa}

A revisão de literatura apresentada mostra que há, atualmente, grande interesse dos pesquisadores da área de avaliação de treinamento nos efeitos de características da clientela e de ambiente organizacional sobre a eficácia de treinamento. Uma particularidade da literatura nacional na área de T\&D é a escassez de estudos que buscam relacionar variáveis individuais com impacto do treinamento no trabalho (Abbad et al.; 2003; Borges-Andrade \& Abbad; 1996). Borges-Andrade e Abbad (1996) e Sallorenzo (2000) consideram importantíssima a incorporação dessas variáveis nos modelos de avaliação de treinamento e 
enfatizam a necessidade de investigar, nos contextos de T\&D, características da clientela, tais como auto-eficácia, locus de controle, bem como outras advindas da Psicologia Social.

Tendo em vista a necessidade de continuar explorando com profundidade o poder explicativo das variáveis individuais (características da clientela) e do suporte à transferência sobre o impacto de treinamento, esta pesquisa teve este objetivo: investigar as contribuições do Autoconceito Profissional e do Suporte à Transferência na explicação da variabilidade de respostas aos instrumentos de auto e heteroavaliação de Impacto do Treinamento no Trabalho. Espera-se que indivíduos que apresentam Autoconceito Profissional elevado e disponham de ambiente organizacional favorável ao uso das novas habilidades percebam de forma mais favorável o Impacto do Treinamento no Trabalho do que aqueles que apresentam baixo Autoconceito Profissional e que se encontrem em ambiente organizacional desfavorável.

\section{Método}

Foram estabelecidas, por meio da Universidade de Brasília, duas parcerias. A primeira foi firmada com a INFRAERO, empresa pública, vinculada ao Ministério da Defesa, que tem como missão atender às necessidades da sociedade relativas a sistemas, produtos e serviços de infra-estrutura aeroportuária e aeronáutica, primando pela qualidade, competitividade e rentabilidade. A segunda parceria foi firmada com os Correios - ECT, cuja teia de serviços e atendimentos se espalha por todo o território. Além de receber e despachar correspondências, a ECT promove serviços de apoio à população, tendo quase a totalidade dos brasileiros como clientes, na condição de remetentes, destinatários ou usuários de serviços.

\section{Amostra de Participantes}

Participaram da pesquisa os funcionários dos Correios e da Infraero que estavam em treinamento durante o período de coleta de dados. A pesquisa realizou-se em duas etapas: na primeira, a coleta de dados foi presencial e na segunda, foi via correio ou e-mail. Como não há garantia de devolução na segunda etapa, o número de participantes ficou bastante reduzido. Além disso, como esta pesquisa exige um pareamento dos questionários aplicados nas duas etapas, o número de participações sofreu nova redução. Desta forma, optou-se por manter duas amostras diferentes, a fim de aproveitar ao máximo o número de participantes. A primeira amostra (auto-avaliação de Impacto como variável critério), foi composta por 117 treinandos dos Correios e da Infraero; e a segunda, 
(heteroavaliação de Impacto como variável critério), por 100 treinandos dos Correios que responderam aos questionários Autoconceito Profissional, autoavaliação de Impacto em Amplitude do Treinamento no Trabalho e Suporte à Transferência tal como percebido pelo treinando. Como estas amostras não apresentam diferenças relevantes entre elas, as seguintes características demográficas e funcionais podem ser citadas de forma geral: a maioria dos treinandos é do sexo masculino, tem entre 21 e 30 anos, trabalha na área fim da empresa, possui cargo de nível básico, tem $2^{\circ}$ grau completo, é casada, possui filhos, é católica e não é praticante de religião.

\section{Instrumentos de Medida}

Foram utilizados os seguintes instrumentos: Escala de Autoconceito Profissional (Tamayo, 2002), composta por 29 itens divididos em 4 fatores; Suporte à Transferência (Abbad, 1999), constituída por 22 itens divididos em dois fatores; e Impacto em Amplitude do Treinamento no Trabalho (Abbad, 1999), composta por um fator único de 12 itens. Os itens das três escalas foram medidos, utilizandose uma escala de 5 pontos do tipo Likert. Os Dados Demográficos foram coletados junto à Escala de Autoconceito Profissional. Para as avaliações de impacto do treinamento no trabalho e suporte à transferência, também foram utilizadas medidas de heteroavaliação (chefias). O Quadro 1 fornece informações gerais sobre os instrumentos utilizados e sua respectiva relação com os componentes do modelo proposto.

\section{Quadro 1: Informações Gerais sobre os Instrumentos Utilizados na Pesquisa}

\begin{tabular}{llc}
\hline \hline \multicolumn{2}{c}{ COMPONENTE 1-AUTOCONCEITO PROFISSIONAL } \\
\hline \hline QUESTIONÁRIO & FATORES & $\mathrm{N}^{\circ}$. DE ITENS \\
1. Escala de Autoconceito Profissional & Segurança Pessoal & 9 \\
& Realização Profissional & 5 \\
& Saúde no Trabalho & 7 \\
& Competência no Trabalho & 8 \\
& Dados Demográficos & 7 \\
\hline \hline
\end{tabular}

\begin{tabular}{llc}
\hline \hline \multicolumn{3}{c}{ COMPONENTE 2 - SUPORTE À TRANSFERENCIA } \\
\hline \hline $\begin{array}{l}\text { QUESTIONÁRIOS } \\
\begin{array}{l}\text { 2. Suporte à Transferência (percebido } \\
\text { pelo treinando e pela chefia) }\end{array}\end{array}$ & $\begin{array}{l}\text { FATORES } \\
\text { Suporte Psicossocial (fatores situacionais de } \\
\text { apoio e conseqüências associadas ao uso } \\
\text { de novas habilidades) } \\
\text { Suporte Material }\end{array}$ & $\begin{array}{c}\mathrm{N}^{\mathrm{O}} \text {. DE ITENS } \\
\text { COMPONENTE 3 - IMPACTO DO TREINAMENTO }\end{array}$ \\
\hline \hline QUESTIONÁRIOS & FATORES & 6 \\
$\begin{array}{l}\text { 3. Impacto do Treinamento no } \\
\text { Trabalho (auto e heteroavaliação) }\end{array}$ & Impacto do Treinamento no Trabalho & $\mathrm{N}^{\circ}$. DE ITENS \\
\hline \hline
\end{tabular}


Os instrumentos utilizados foram submetidos, inicialmente, à análise dos Componentes Principais (PC) com o objetivo de verificar o grau de fatorabilidade dos dados e estimar o número de componentes. Os critérios adotados para a identificação do número de fatores foram: o valor dos eigenvalues (maiores ou iguais a um), a importância do fator (critério de 3\% da variância explicada pelo fator) e o teste do scree plot. Para a extração dos fatores, prosseguiu-se com a análise de Fatoração dos Eixos Principais (PAF) com supressão de itens que apresentam cargas fatoriais inferiores a 0,30 e tratamento pairwise dos casos omissos e rotação ortogonal (varimax). A análise de consistência interna (Alpha de Cronbach) dos itens foi aplicada aos fatores resultantes. Essas análises permitiram validar estatisticamente os instrumentos e julgar a sua confiabilidade.

\section{Coleta e Análise de Dados}

A coleta de dados se deu em dois momentos distintos: 1) antes do treinamento, quando foi aplicada, de forma coletiva, a Escala de Autoconceito Profissional acoplada com o questionário de dados demográficos; e 2) três meses após o término do treinamento, quando foram enviados aos participantes e suas respectivas chefias, via malote ou correio eletrônico, os instrumentos de Suporte à Transferência e Impacto do Treinamento no Trabalho.

As respostas dos participantes aos questionários de Autoconceito Profissional, Suporte à Transferência e Impacto do Treinamento no Trabalho foram pareadas de forma a possibilitar o teste do modelo. Para a execução das análises estatísticas, utilizou-se o Programa Estatístico SPSS (Statistical Package for the Social Science), versão 10.0.

Inicialmente, os dados foram submetidos a análises exploratórias e descritivas e, em seguida, a análises de regressão múltipla padrão e hierárquicas. Estas foram realizadas com o intuito de compreender o relacionamento existente entre as variáveis antecedentes e a variável critério, verificando o grau de predição das mesmas. O método de regressão hierárquico foi utilizado para testar hipóteses advindas das primeiras análises.

\section{Resultados}

Dois modelos de regressão múltipla foram utilizados (Tamayo, 2002): no primeiro, a variável critério foi a auto-avaliação de impacto do treinamento no trabalho (aait); e no segundo, a heteroavaliação de impacto do treinamento no trabalho (hait). As variáveis antecedentes foram as seguintes: autoconceito/ 
segurança pessoal (acsp), autoconceito/realização profissional (acrp), autoconceito/saúde no trabalho (acst), autoconceito/competência no trabalho (acct), suporte psicossocial à transferência percebido pelo treinando (sptt) e pela chefia (sptc), suporte material à transferência percebido pelo treinando(smtt), e pela chefia (smtc). Vale ressaltar que as variáveis antecedentes: suporte psicossocial e material à transferência percebido pelos participantes (sptt e smtt) e pelas chefias (sptc e sptm) foram consideradas apenas nos modelos de regressão, cujas variáveis critério eram, respectivamente, auto e heteroavaliação do impacto do treinamento no trabalho.

As variáveis incluídas nos modelos de análise foram submetidas a análises exploratórias. Nenhuma delas apresentou mais de 5\% de valores ausentes. Com a finalidade de otimizar o aproveitamento dos casos, optou-se pelo tratamento pairwise para dados omissos, tanto na regressão múltipla padrão quanto na hierárquica. $\mathrm{O}$ cálculo da distância Mahalanobis $(\alpha=0,001)$ possibilitou a identificação de um caso extremo multivariado no arquivo de dados de autoavaliação de Impacto, sendo este excluído.

A primeira análise de regressão múltipla padrão foi realizada tendo como variável critério a auto-avaliação de Impacto. As variáveis antecedentes foram os quatro fatores de Autoconceito Profissional e os dois fatores de Suporte à Transferência percebido pelo treinando. $\mathrm{O}$ coeficiente de regressão $\mathrm{R}$ foi significativamente diferente de zero, $\mathrm{F}(6,11)=9,15, \mathrm{p}<0,001$. Para o coeficiente de regressão, que diferiu significativamente de zero, limites de confiança de $95 \%$ foram calculados. $\mathrm{O}$ coeficiente, Suporte Psicossocial à Transferência percebido pelo treinando, apresentou um intervalo de confiança variando entre 0,33 e 0,62. A Tabela 1 apresenta as correlações entre as variáveis, a média (X), o desvio padrão (DP), os coeficientes de regressão não padronizados (B), a constante, os coeficientes de regressão padronizados $(\beta)$, as correlações semiparciais $(\mathrm{Sr} 2)$, bem como o R, o R2 e o R2 ajustado.

\section{Tabela 1: Regressão Múltipla Padrão com Variável Critério Auto- Avaliação de Impacto}

\begin{tabular}{|c|c|c|c|c|c|c|c|c|c|c|}
\hline VAR & $\begin{array}{l}\text { Aimp } \\
\text { (VD) }\end{array}$ & Acsp & Acrp & Acst & Acct & Sptt & Smtt & $\mathrm{B}$ & $\beta$ & $\mathrm{Sr}^{2}$ \\
\hline Acsp & $-0,14$ & & & & & & & 0,08 & $-0,08$ & 0,00 \\
\hline Acrp & 0,16 & $-0,13$ & & & & & & $-0,02$ & $-0,03$ & 0,00 \\
\hline Acst & $-0,07$ & 0,14 & $-0,36$ & & & & & $-0,01$ & $-0,01$ & 0,00 \\
\hline Acct & 0,16 & $-0,09$ & 0,15 & $-0,20$ & & & & 0,32 & 0,13 & 0,01 \\
\hline Sptt & 0,56 & $-0,09$ & $\mathbf{0 , 3 0}$ & $-0,07$ & 0,05 & & & $0,47 *$ & $\mathbf{0 , 5 7}$ & 0,25 \\
\hline Smtt & 0,16 & 0,07 & 0,17 & $-0,13$ & 0,01 & 0,37 & & $-0,03$ & $-0,04$ & 0,00 \\
\hline $\mathrm{X}$ & 3,70 & 2,43 & 3,70 & 1,76 & 4,76 & 3,19 & 3,44 & & & \\
\hline DP & 0,70 & 0,63 & 0,84 & 0,57 & 0,27 & 0,84 & 1,01 & & & \\
\hline \multicolumn{3}{|c|}{ Constante $=1,05$} & \multicolumn{2}{|c|}{$\mathrm{R}^{2}=0,33$} & & \multicolumn{2}{|c|}{$\mathrm{R}^{2}$ (ajust) $=0,30$} & \multicolumn{2}{|c|}{$\mathrm{R}=0,58$} & \\
\hline
\end{tabular}


Entre as seis variáveis antecedentes utilizadas, apenas uma contribuiu significativamente para explicar a auto-avaliação de Impacto do Treinamento no Trabalho: Suporte Psicossocial percebido pelo treinando $(\mathrm{sr} 2=0,25)$. O Beta positivo desta variável indica que, quanto maior a percepção de Suporte Psicossocial por parte dos treinandos, maior a percepção, também por parte dos treinandos, de Impacto do Treinamento no Trabalho. Combinadas, as outras cinco variáveis antecedentes contribuem em apenas $5 \%$ da variabilidade compartilhada. O conjunto das seis variáveis explicam 33\% (30\% ajustado) da variância total do Impacto do Treinamento no Trabalho segundo a percepção dos treinandos.

A segunda análise de regressão múltipla padrão realizou-se tendo como variável critério a heteroavaliação de Impacto. As variáveis antecedentes foram os quatro fatores de Autoconceito Profissional e os dois fatores de Suporte à Transferência percebido pela chefia. $O$ coeficiente de regressão $R$ foi significativamente diferente de zero, $F(6,93)=4,42, p<0,001$. Para o coeficiente de regressão, que diferiu significativamente de zero, calcularam-se limites de confiança de $95 \%$. O coeficiente, Suporte Material à Transferência percebido pela chefia do treinando, apresentou um intervalo de confiança variando entre 0,16 e 0,41. A Tabela 2 apresenta as correlações entre as variáveis, a média (X), o desvio padrão (DP), os coeficientes de regressão não padronizados (B), a constante, os coeficientes de regressão padronizados $(\beta)$, as correlações semiparciais $(\mathrm{Sr} 2)$, bem como o R, o R2 e o R2 ajustado.

\section{Tabela 2: Regressão Múltipla Padrão com Variável Critério Heteroavaliação de Impacto}

\begin{tabular}{|c|c|c|c|c|c|c|c|c|c|c|}
\hline VAR & $\begin{array}{l}\text { Aimp } \\
\text { (VD) }\end{array}$ & Acsp & Acrp & Acst & Acct & Sptc & Smtc & B & $\beta$ & $\overline{\mathrm{Sr}^{2}}$ \\
\hline Acsp & $-0,12$ & & & & & & & $-0,15$ & $-0,18$ & 0,03 \\
\hline Acrp & 0,16 & 0,14 & & & & & & 0,08 & 0,16 & 0,02 \\
\hline Acst & $-0,05$ & 0,05 & $-0,28$ & & & & & 0,02 & 0,04 & 0,00 \\
\hline Acct & $-0,02$ & $-0,05$ & 0,17 & $-0,11$ & & & & $-0,12$ & $-0,07$ & 0,00 \\
\hline Sptc & 0,10 & 0,14 & 0,16 & $-0,19$ & 0,03 & & & 0,02 & 0,02 & 0,00 \\
\hline Smtc & 0,42 & 0,07 & 0,09 & $-0,08$ & 0,03 & 0,21 & & $0,29 *$ & 0,42 & 0.16 \\
\hline $\mathrm{X}$ & 3,45 & 2,59 & 3,66 & 2,00 & 4,75 & 3,80 & 3,53 & & & \\
\hline DP & 0,49 & 0,60 & 0,90 & 0,72 & 0,26 & 0,50 & 0,71 & & & \\
\hline \multicolumn{3}{|c|}{ Constante $=2,95$} & \multicolumn{3}{|c|}{$\mathrm{R}^{2}=0,22$} & \multicolumn{2}{|c|}{$\mathrm{R}^{2}$ (ajust) $=0,17$} & \multicolumn{3}{|c|}{$\mathrm{R}=0,47$} \\
\hline
\end{tabular}

Entre as seis variáveis antecedentes utilizadas, apenas uma contribuiu significativamente para explicar a heteroavaliação de Impacto do Treinamento no Trabalho: Suporte Material percebido pela chefia dos treinando $(\mathrm{sr} 2=0,16)$. O Beta positivo desta variável indica que, quanto maior a percepção das chefias do Suporte Material, maior a percepção também de Impacto do Treinamento. Combinadas, as outras cinco variáveis antecedentes contribuem em $1 \%$ da 
variabilidade compartilhada. O poder de predição das seis variáveis agrupadas é de $22 \%$ (17\% ajustado) da variabilidade de Impacto do Treinamento no Trabalho, segundo a percepção das chefias.

$\mathrm{Na}$ tentativa de compreender o porquê das variáveis de Autoconceito Profissional não estarem contribuindo significativamente para a explicação da percepção, tanto pelo treinando quanto pela chefia, do Impacto do Treinamento no Trabalho, optou-se pela realização de análises de regressão múltipla hierárquicas para cada modelo. A hipótese era de que o Autoconceito Profissional poderia ser variável moderadora do relacionamento entre Suporte à Transferência e Impacto do Treinamento no Trabalho (Tamayo, 2002). Quando determinada variável $(Z)$ é identificada como moderadora, significa que a relação entre a variável antecedente $(\mathrm{X})$ e a variável critério $(\mathrm{Y})$ depende do valor da variável moderadora (Z), isto é, a influência da variável (X) sobre (Y) não será a mesma para todos os valores de $(Z)$.

Para testar a moderação do Autoconceito Profissional, calculou-se a interação entre cada um dos seus fatores e os de suporte. É uma alteração significativa no índice de mudança do R2 após a inclusão dessas variáveis de interação na regressão que indica se existe moderação da variável testada. A moderação da variável Autoconceito Profissional foi testada para os dois modelos de regressão. As variáveis antecedentes foram agrupadas e inseridas na análise na seguinte ordem de entrada: 1) os dois fatores de Suporte à Transferência; 2) os quatro fatores de Autoconceito Profissional; e 3) as oito variáveis de interação de Autoconceito Profissional e Suporte.

Após a inclusão das variáveis de interação na regressão não houve alteração significativa no índice de mudança do R2, indicando que a variável de Autoconceito Profissional não modera a relação entre Suporte à Transferência e Impacto do Treinamento no Trabalho. Optou-se então por testar, separadamente, a moderação de cada um dos fatores de Autoconceito Profissional, mas os resultados encontrados não foram diferentes dos anteriores. Em outras palavras, a influência do Suporte à Transferência sobre o Impacto do Treinamento no Trabalho é independente do nível de Autoconceito Profissional (ou de qualquer um de seus fatores) apresentado pelo treinando.

\section{Discussão}

Considerando que o objetivo deste estudo é investigar o relacionamento entre as variáveis antecedentes Autoconceito Profissional e Suporte à Transferência 
e a variável critério Impacto do Treinamento no Trabalho, os resultados demonstraram que apenas a variável de Suporte é preditora de Impacto de Treinamento.

Quando a variável critério foi auto-avaliação de Impacto do Treinamento no Trabalho, verificou-se o forte poder preditivo $(s r 2=0,25)$ da variável Suporte Psicossocial à Transferência, o que corrobora os dados encontrados na literatura (Abbad, 1999; Abbad et al., 2003; Roullier \& Goldstein, 1993; Salas \& CannonBowers, 2001; Sallorenzo, 2000). A contribuição do Suporte Psicossocial à Transferência para a predição de Impacto do Treinamento no Trabalho já é bastante conhecida e enfatizada na literatura científica da área. Treinandos que percebem o suporte em seu ambiente de trabalho de forma favorável também relatam melhoria em seus desempenhos após a participação em treinamentos.

Quando a variável critério foi a heteroavaliação de Impacto do Treinamento no Trabalho, a única variável antecedente capaz de contribuir de forma significativa para a explicação do Impacto foi o Suporte Material $(s r 2=0,16)$. Este resultado é interessante pelo fato de os chefes dos treinandos considerarem que o Impacto de Treinamento depende do um tipo de suporte externo e, até certo ponto, distante deles. Esse resultado confirma os do único estudo encontrado (Borges-Andrade, Morandini, \& Machado, 1999), no qual a variável Suporte Material foi preditora de Impacto de Treinamento. Chefes que percebem o Suporte Material fornecido pela organização de forma favorável percebem também melhoria no desempenho e nas atitudes de seus colaboradores após a participação em treinamentos.

Isto reforça o fato de que o Suporte à Transferência é necessário para que haja Impacto do Treinamento no Trabalho. As organizações precisam fazer uma avaliação séria do suporte fornecido para seus empregados antes de submetêlos a treinamentos que podem não surtir efeito, caso não haja qualidade no delineamento do contexto pós-treinamento.

Apesar de este estudo ter surgido principalmente em função da necessidade de avaliar a contribuição de variáveis individuais na predição de Impacto do Treinamento no Trabalho, os resultados mostraram que a variável Autoconceito Profissional não prediz Impacto. O modelo hipotético, testado mediante análise de regressão múltipla hierárquica, que sugeria moderação do autoconceito sobre a relação entre Suporte à Transferência e Impacto do Treinamento no Trabalho tampouco foi confirmado.

Porém ainda é prematuro concluir que o Autoconceito Profissional não está relacionado com Impacto do Treinamento no Trabalho. Como já foi mencionado anteriormente, o autoconceito refere-se a aspectos disposicionais de natureza dinâmica, que se modificam em função das experiências do indivíduo (Cerqueira, 
1991). Desta forma, o procedimento de coleta de dados, utilizado na pesquisa, pode não ter sido o mais adequado para a investigação do relacionamento entre Autoconceito Profissional e Impacto, visto que a medida de Autoconceito ocorreu antes do treinamento. O Autoconceito Profissional é afetado por vários fatores e, provavelmente, eventos instrucionais como os treinamentos avaliados neste estudo, são capazes de alterar a forma como o indivíduo se percebe em relação ao seu trabalho e às tarefas que executa. Assim, é possível que os resultados fossem diferentes, se a medida ocorresse algum tempo após o termino do mesmo, e fosse aplicada simultaneamente com a medida de Suporte à Transferência e Impacto do Treinamento no Trabalho. Um indivíduo que passou por um curso de capacitação talvez apresente aumento no nível de Autoconceito Profissional, o que pode afetar a transferência, bem como seu desempenho no cargo.

Desta forma, sugere-se a realização de novos estudos que integrem o Autoconceito Profissional como variável explicativa de Impacto, utilizando delineamento de pesquisa com a mensuração do Autoconceito após o término do treinamento. Poder-se-ia ainda efetuar duas medidas de Autoconceito: uma antes e a outra após o treinamento (pré e pós-teste com grupo controle). Neste caso, a primeira medida, que se referiria à forma como o indivíduo se percebia antes de ser treinado, serviria apenas para verificar se houve alteração no Autoconceito Profissional do indivíduo pelo fato deste ter passado por um treinamento.

Além disso, talvez os treinamentos avaliados não tenham tido efeito significativo sobre o Autoconceito Profissional do treinando, já que a grande maioria deles não ultrapassava 40 horas e treinava habilidades específicas em salas de aula. Talvez o poder preditivo do autoconceito seja mais importante quando relacionado a planos de carreira. Muitos executivos vêm investindo na abertura de universidades corporativas como meio de intensificar o desenvolvimento dos empregados. Segundo Eboli (1999), a universidade corporativa tem como missão formação e desenvolvimento de talentos humanos na gestão dos negócios, promovendo gestão do conhecimento organizacional (geração, assimilação, difusão e aplicação), por meio de um processo de aprendizagem ativa e contínua. $\mathrm{O}$ principal objetivo de uma universidade corporativa é o desenvolvimento de competências críticas em vez de habilidades, buscando aumentar o desempenho do indivíduo e não apenas suas habilidades. Na medida em que a educação corporativa está trabalhando com currículos em vez de cursos isolados, o estudo do Autoconceito Profissional pode ser útil para explicar a aquisição desses tipos de competências e disposições individuais.

Para Eboli (1999), a postura voltada à aprendizagem contínua é um processo de constante crescimento e fortalecimento dos indivíduos. Cursos isolados talvez não cheguem a mudar o Autoconceito Profissional; mas vale a pena investigar 
se uma educação contínua teria algum efeito sobre a variável, já que um trabalho mais abrangente com os indivíduos pode mudar o status destes e, conseqüentemente suas auto-avaliações. Assim, considera-se que o Autoconceito Profissional pode ser uma variável promissora para o estudo do planejamento individual de carreira.

Embora ainda distante de responder a todos os questionamentos da área de avaliação de treinamento, este trabalho aponta na direção de que, como atestaram vários autores da literatura nacional e internacional, não há mais como desprezar o fato de que o Suporte Psicossocial à Transferência é o grande responsável pela explicação de Impacto do Treinamento no Trabalho. Desta forma, é inevitável e essencial investir em pesquisas capazes de identificar obstáculos à transferência de treinamento no trabalho e investigar diferentes estratégias pós-treinamento que possam maximizar o uso das novas habilidades adquiridas.

\section{Artigo recebido em 04.05.2004. Aprovado em 17.08.2004.}

\section{ReferênCIAS Bibliográficas}

Abbad, G. (1999).

Um modelo integrado de avaliação do impacto do treinamento no trabalho - IMPACT. Tese de Doutorado, Instituto de Psicologia, Universidade de Brasília, DF, Brasil.

Abbad, G.,

Gama, A. L., \&

Borges-Andrade, J. E. (2000, setembro/ dezembro).

Treinamento: análise do relacionamento da avaliação nos níveis de reação, aprendizagem e impacto no trabalho. Revista de Administração Contemporânea, 4(3), 25-45.

Abbad, G.,

Pilati, R., \&

Pantoja, M. J. (2003, julho/setembro).

Avaliação de treinamento: análise de literatura e agenda de pesquisa. Revista de Administração da Universidade de São Paulo, 38(3), 205-218.

Abbad, G., \&

Sallorenzo, L. H. (2001, abril/junho).

Desenvolvimento e validação de escalas de suporte à transferência de treinamento. Revista de Administração, 36(2), 33-45.

Alliger, G. M., \& Janak, E. A. (1989, Summer).

Kirkpatrick's levels of training criteria: thirty years later. Personnel Psychology, 42(2), 331-342.

Bastos, A. V. B.

(1991, julho/dezembro). Validação da escala locus de controle no trabalho. PSICO, 22(2), 133-154. 
Borges-Andrade, J. E. (1982, junho). Avaliação somativa de sistemas instrucionais: integração de três propostas. Tecnologia Educacional, 11(46), 29-39.

Borges-Andrade, J. E., \&

Abbad, G. (1996, abril/junho).

Treinamento e desenvolvimento: reflexões sobre suas pesquisas científicas. Revista de Administração, 31(2), 112-125.

Borges-Andrade, J. E.,

Morandini, D. C., \&

Machado, M. S. (1999).

Impacto de treinamento gerencial e efetividade de equipes em ambiente de inovação tecnológica [Resumo]. Anais da Reunião Anual de Psicologia, Ribeirão Preto, SP, 29.

Britto, M. J. P. (1999).

Avaliação de impacto de treinamento na área de reabilitação: preditores individuais e situacionais. Dissertação de Mestrado, Universidade de Brasília, DF, Brasil.

Cerqueira, T. C. S. (1991).

Possíveis influências do autoconceito e do locus de controle sobre o rendimento acadêmico. Dissertação de Mestrado, Universidade de Brasília, DF, Brasil.

Costa, P. C. G. (1996).

Relações entre o autoconceito e a satisfação no trabalho de funcionários de uma instituição bancária. Dissertação de Mestrado, Universidade de Brasília, DF, Brasil.

Cronbach, L. J., \&

Snow, R. E. (1977).

$$
\text { Aptitudes and instructional }
$$

methods: a handbook for research on interactions. New York: Irvington.

Eboli, M. P. (1999, outubro/dezembro). Universidade corporativa: ameaça ou oportunidade para as escolas tradicionais de administração? Revista de Administração, 34(4), 5664.

Goldstein, I. L. (1991).

Training in work organizations. In M. D. Dunnette, \& L. M. Hough (Eds.). Handbook of industrial and organizational psychology. (2nd ed., pp. 507-619). Palo Alto, Cal.: Consulting Psychologists.

Hamblin, A. C. (1978).

Avaliação e controle do treinamento. São Paulo: McGraw-Hill do Brasil.

Kihlsrom, J. F., \&

Cantor, S. E. (1984).

Mental representations of the self. In L. Berkowitz (Ed.). Advances in Experimental Social Psychology (Vol. 21, pp. 145-180). New York: Academic Press.

Kirkpatrick, D. L. (1976).

Evaluation of training. In R. L. Craig (Org.). Training and development handhook (2nd ed., pp. 18.1-18.27.). New York: Mc Graw-Hill.

L'Ecuyer, R. (1978).

Le concept de soi. Paris: Presses Universitaires de France.

Lummertz, J. G., \& Biaggio, A. M. B. (1986, abril/junho).

Relações entre autoconceito e nível de satisfação familiar em adolescentes. Arquivos Brasileiros de Psicologia, 38(2), 158-166. 
Nadler, L. (1984).

The handbook of human resources development. New York: Wiley.

Nezlek, J. B., \&

Plesko, R. M. (2001, February).

Day-to-day relationships among selfconcept clarity, self-esteem, daily events and mood. Personality and Social Psychology Bulletin, 27(2), 201-211.

Roullier, J. Z., \&

Goldstein, I. L. (1993, October/December) The relationship between organizational transfer climate and positive transfer of training. Human Resource Development Quarterly, 4(4), 377-390.

Salas, E., \&

Cannon-Bowers, J. A. (2001, Febuary). A The science of training: a decade of progress. Annual Review of Psychology, 52, pp. 471-499.

Sallorenzo, L. H. (2000).

Avaliação de impacto de treinamento no trabalho: analisando e comparando modelos de predição. Dissertação de Mestrado, Universidade de Brasília, DF, Brasil.

Sedikides, C., \&

Skowronski, J. J. (1997, January/March). The symbolic self in evolutionary context. Personality and Social Psychology Review, 1(1), 80-102.

Shavelson, R. J.,

Hubner, H., \&

Stanton, G. C. (1976, Summer).

Self-concept: validation of construct interpretations. Review of Educational Research, 46(3), 407441.
Tamayo, A. (1982, abril/junho).

Autoconceito e região de origem. Arquivos Brasileiros de Psicologia, 34(2), 60-64.

Tamayo, A. (1993).

Autoconcepto y prevención. In J. R. Rojas (Ed.). Quinta antología: salud y adolescencia (pp. 18-28.). San José de Costarica: Caja Costarricense de Seguro Social.

Tamayo, N. (2002).

Autoconceito profissional, suporte à transferência e impacto do treinamento no trabalho. Dissertação de Mestrado, Universidade de Brasília, DF, Brasil.

Tannenbaum, S. I.,

Mathieu, J. E.,

Salas, E., \&

Cannon-Bowers, J. A. (1991, December).

Meeting trainee's expectations: the influence of training fulfillment on the development of commitment, selfefficacy and motivation. Journal of Applied Psychology, 76(6), 759-769.

Tannenbaum, S. I., \&

Yukl, G. (1992, January).

Training and development in work organizations. Annual Review of Psychology, 43, pp. 399-441.

Vargas, M. R. M. (1996, abril/junho).

Treinamento e desenvolvimento: reflexões sobre seus métodos. Revista de Administração, 31(2), 126136. 